\title{
Multiple resource limitation of plants and its consequences for herbivores and their impacts
}

\author{
Neha Mohanbabu ${ }^{1}$ and Mark Ritchie ${ }^{2}$ \\ ${ }^{1}$ Syracuse University College of Arts and Sciences \\ ${ }^{2}$ Syracuse University
}

November 9, 2020

\begin{abstract}
Substantial evidence suggests plants and herbivores are limited by multiple resources but their role in driving plant-herbivore interactions is still poorly understood. Here we model multiple resource limitation of plants and herbivores and derive analytical solutions for steady-state plant biomass, herbivore biomass and herbivore impact. The model predicts "apparent" limitation of herbivore biomass by resources that otherwise do not limit herbivore growth. Consequently, higher supply of plant-growth limiting resources allows herbivores to persist at lower supplies of herbivore growth-limiting resources. Likewise, increased supply of these non-limiting resources to herbivores can dramatically increase herbivore impacts on plants. Additionally, the outcomes of herbivore exclusion experiments should differ along different resource gradients, depending on herbivore response to plant resource concentrations. Analysis of existing and new data from marine, freshwater and terrestrial systems supports several of these predictions. Our analysis expands ecologists' understanding of plant-herbivore dynamics to accommodate multiple limiting resources.
\end{abstract}

\section{Hosted file}

Mohanbabu_MRL_herbivory_final.pdf available at https://authorea.com/users/374337/articles/ 491877-multiple-resource-limitation-of-plants-and-its-consequences-for-herbivores-andtheir-impacts 